\title{
A Hybrid PSO-Fuzzy Model for Determining the Category of 85th Speed
}

\author{
Abbas Mahmoudabadi ${ }^{1}$ and Ali Ghazizadeh ${ }^{2}$ \\ ${ }^{1}$ Department of Industrial Engineering, Payame Noor University (PNU), Shahnaz Alley, Nourian Street, North Dibagi Avenue, \\ Tehran, Iran \\ ${ }^{2}$ Technical and Engineering Department, Payame Noor University (PNU), Firouzbakhsh Street, Movahed Danesh Avenue, \\ Aqdasieh, Tehran, Iran \\ Correspondence should be addressed to Abbas Mahmoudabadi; mahmoudabadi@phd.pnu.ac.ir
}

Received 22 March 2013; Accepted 21 May 2013

Academic Editor: Ling Wang

Copyright (C) 2013 A. Mahmoudabadi and A. Ghazizadeh. This is an open access article distributed under the Creative Commons Attribution License, which permits unrestricted use, distribution, and reproduction in any medium, provided the original work is properly cited.

\begin{abstract}
The 85th speed of vehicles is one of the traffic engineering parameters used by road safety equipment designers. It is usually used for maintenance activities and designing of warning signs and road equipments. High measuring costs of speed data collection lead decision makers to define a methodology for determining the category of 85th speed using indirect parameters. In this research work, focusing on undivided intercity roads, a hybrid particle-swarm-optimization- (PSO-) fuzzy model has been developed to determine the category of 85 th speed. In this model, geometric design parameters including roads' width and length characteristics and roadside land use are considered as input variables whereas the category of the 85th speed is output variable. A set of experimental data is used for evaluating the performance of the proposed model comparing to a well-known model of exponential regression. It is shown that the developed PSO-fuzzy model is capable of determining the category of 85th speed with an accuracy of $96 \%$, while exponential regression can estimate that with up to $84 \%$ accuracy. Variable effectiveness procedure shows that the lane width has more direct effect on 85th speed than shoulder width and the number of access points. The percentage of forbidden overtaking is also found to have indirect effect on 85 th speed.
\end{abstract}

\section{Introduction}

The 85th speed is one of the most important traffic engineering parameters. By definition, the 85th speed is the maximum speed that eighty-five percent (\%85) of drivers prefer to drive with a speed less than that. The importance of this parameter is that the majority of drivers drive within their safe considered speed, so the 85th speed is the maximum speed considered as safe speed by most of drivers [1]. The 85th speed is being used in some technical applications such as designing of road safety infrastructure, planning road maintenance programs, and setting warning signs, while construction workers are working on the road in order to provide the traffic with safe passage. Many parameters affect on 85th speed including authorized speed, traffic volume, land topography, and roadside land use condensations [1]. Traditionally, 85th speed is calculated using vehicle's speed on a certain road. Many techniques have been utilized for estimating vehicles' speed, mainly need for electronic devices and infrastructures. Image processing technique [2], combination of fuzzy logic and image processing [3], traffic counting and occupancy data [4], vehicle tracking [5], optical sensors [6], RF-based vehicle detection [7], the field of mechanical engineering by kinematics relationship of measured signals [8], and traffic survey [1] are the well-known techniques on speed data collection. Expensive devices are needed for utilizing either of the previously mentioned techniques. Therefore, developing a low-cost procedure to estimate the category of 85 th speed is quite interesting.

Although 85th speed is a measurable parameter, its exact quantity does not play significant role in real applications. So, in the literature, researchers utilized linguistic variables 
for this kind of parameters. For example, traffic volume is measured precisely; however, it is usually expressed by linguistic words such as heavy, normal, or low traffic $[9,10]$. Therefore, it is considerable to utilize fuzzy variables in order to represent traffic parameters corresponding to the 85th speed.

Fuzzy approaches capture the senses of experts about input parameters and convert them to fuzzy outputs using specified fuzzy relationships between input and output variables. The process of developing a fuzzy model has three stages. The first is to define membership functions corresponding to input and output variables, the second is to define rules, and the third is to adjust input-output relationship [11].

For this advantage of fuzzy approaches, they are utilized in many fields of studies such as energy $[12,13]$, road safety evaluation programs [14], and road control system [15]. In the field of traffic and transportation engineering, many research works have been found in the literature [16]. For example fuzzy approaches have been utilized for developing signal detection theory in order to perceive workers' hazards on subway operations [17], developing fuzzy model to set traffic light timing [18], proposing an expert fuzzy system to predict object collision [19], and assessment of traffic parameters [20]. Fuzzy approaches have also been used to estimate traffic parameters such as traffic jam [10] and passenger car unit [9].

The combinations of fuzzy and heuristic methods have also been used for fuzzy clustering [21], forecasting [22], job scheduling [23], and time series planning [24]. Another application is a combination of fuzzy logic and particle swarm optimization to achieve the best performance of setting fuzzy parameters and improving definition of fuzzy logic relations. For example, a combination of PSO and GA is used in the process of decision making [25], a balanced fuzzy PSO for distinguishing differences between positive and negative information of membership functions [26], an encoding fuzzy model utilized to achieve the structures and parameters [27]. An improved method of optimization using fuzzy logic to integrate the results of particle swarm optimization and genetic algorithm for parameter tuning [28] is also found in this area.

In this research work, a combination of particle swarm optimization and fuzzy approach is used to develop a PSOfuzzy model to determine the category of 85 th speed in intercity roads. The ability of fuzzy approach on defining linguistic variables and PSO ability for parameter tuning are combined in the proposed model, which is capable of determining the category of the 85 th speed by experts' points of view and low cost measures. The main contribution in this paper is developing a model to estimate the category of 85th speed of vehicles using a few measurable factors. This concept can be applied in undivided roads, in which there is no enough electronic devices or equipments to collect reliable data particularly corresponding to speed and traffic volume. After introduction, Section 2 presents a very short description on particle swarm optimization technique. Input and output variables corresponding to the model are defined in Section 3, followed by model development in Section 4. Comparison of the results using the experimental data with a well-known exponential regression method of prediction as well as summary and conclusion will be explained at the next on Sections 5 and 6, respectively.

\section{Particle Swarm Optimization}

One of the well-known evolutionary algorithms used in optimization problems is particle swarm optimization (PSO). Particle swarm optimization (PSO) is a heuristic technique, developed based on bird flocking or fish schooling by Kennedy and Eberhart in 1995 [29]. It is a populationbased technique the same as Genetic Algorithms (GA). Although, PSO's theoretical foundation is rather weak, but its application has been proved to be effective [30]. In PSO, feasible solutions, called particles (individuals), are generated by moving particles towards the current optimum particles. The best solution in the current population is called Pbest and in the whole generation is called Gbest [29]. The main idea in this process is to generate new populations by changing the velocity of each particle towards its Pbest and Gbest locations. Running stages continue until stopping criteria are met. At final stage, Gbest is considered as the best solution. Figure 1 illustrates the overall view of particle swarm optimization.

In PSO, a particle is composed of three vectors of $X, P$, and $V$, which represent the current location, location of the best solution, and a gradient for moving particles, respectively. It also includes two fitness values: $X$-Fitness records the fitness of the $X$-vector and $P$-Fitness records the fitness of $P$-vector. Moving each particle is done by simply adding $V$-vector to the $X$-vector, while $V$-vector must have been adjusted by random generated rates [31]. In each iteration, the positions of particles are updated by using (1), while $V$-vector (velocity) is adjusted by (2)

$$
\begin{gathered}
X i d=X i d+V i d, \\
V i d=V i d+\varphi 1 \times \text { random } \times(P i d-X i d) \\
+\varphi 2 \times \text { random } \times(P g d-X i d),
\end{gathered}
$$

where, $X i d$ is the position of particle, $d$ is dimension, Vid is moving rate, $P$ id is location of the best solution in iteration $i$, and Pgd is the location of global best solution. Different amounts of $\varphi 1$ and $\varphi 2$ identify the types of PSO including full model $(\varphi 1, \varphi 2>0)$, cognition only $(\varphi 1>0, \varphi 2=0)$, social only $(\varphi 1=0, \varphi 2>0)$, and selfless $(\varphi 1=0, \varphi 2>0$ and $g \# i)$ [31].

There are two well-known techniques of controlling velocities called inertia factor and constriction coefficient. When inertia factor is used, (2) is changed to (3) and when constriction coefficient is used (2) is changed to (4) [32]

$$
\begin{array}{r}
\text { Vid }=\omega \times \text { Vid }+\varphi 1 \times \text { random } \times(\text { Pid }-X i d) \\
+\varphi 2 \times \text { random } \times(P g d-X i d), \\
\text { Vid }=K \times[\text { Vid }+\varphi 1 \times \text { random } \times(P i d-X i d) \\
+\varphi 2 \times \text { random } \times(P g d-X i d)],
\end{array}
$$




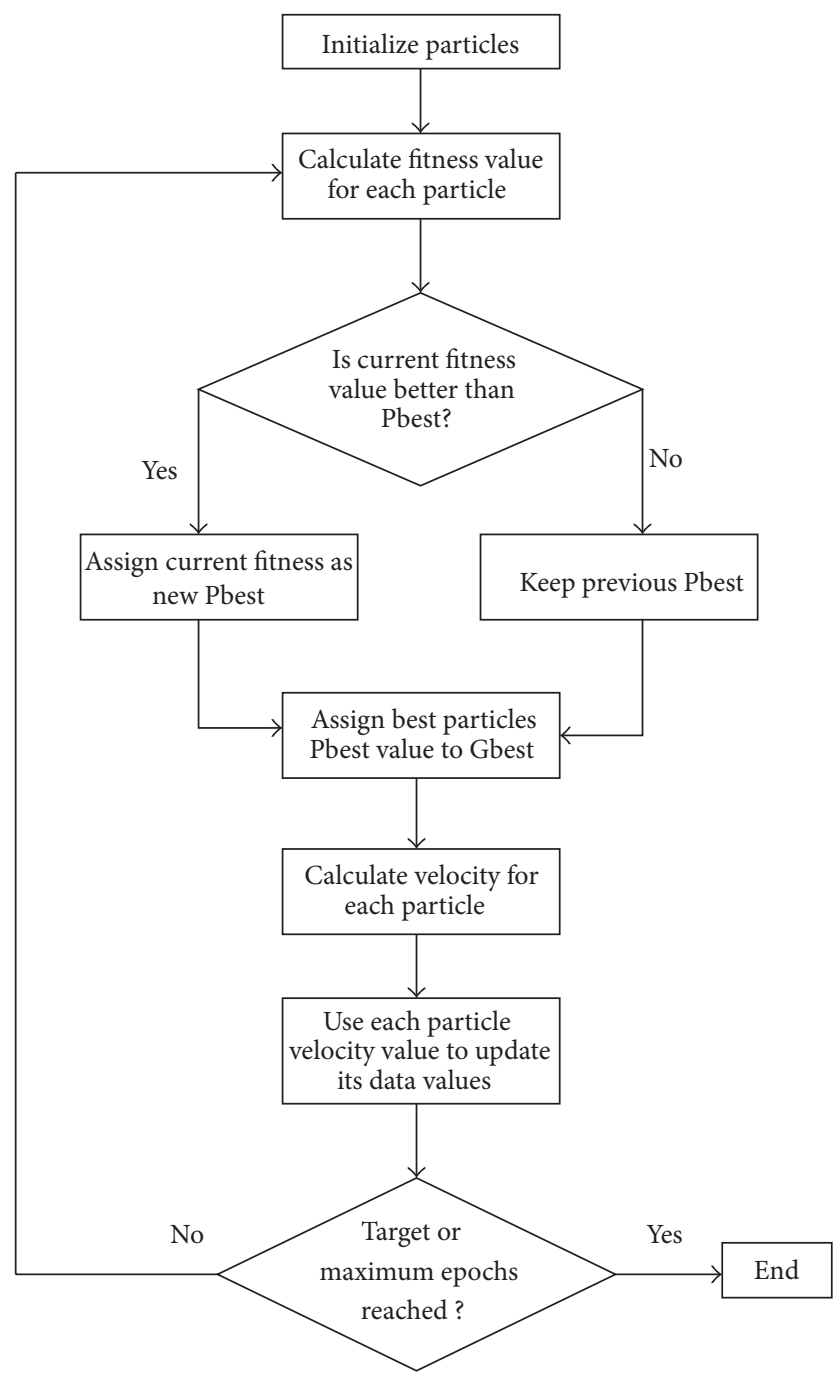

Figure 1: Overall view of particle swarm optimization.

where

$$
K=\frac{2}{\left|2-\varphi-\operatorname{sqrt}\left(\varphi^{2}-4 \times \varphi\right)\right|}, \quad \varphi=\varphi 1+\varphi 2, \varphi>4 .
$$

Swarm topology is another concern in PSO. In general, a fully connected neighborhood topology named Gbest, in which every particle is a neighbor of every other particle, is used for attracting particles to the best solution. However, each particle has access to the information corresponding to its neighborhood according to a certain swarm topology called local neighbor models (Lbest) [33]. The two most common topologies are ring topology, in which each particle is connected with two neighbors, and wheel topology, in which the individuals are isolated from one to another, and all information is communicated to a focal individual [33].

If there is no centralized concept of a global Gbest, particles select each others as informants and swarm topology is highly dependent on the threshold $p$, which is constant for all particles in the swarm [33]. Particles are chosen if random value is less than $p$ and Gbest is selected among the chosen particles. The mentioned process of choosing particles is called stochastic star of swarm topology. Finally at the end of PSO, two methods are used for updating particles: synchronous method, in which newly discovered solutions are used more quickly and asynchronous method in which all solutions have equal chance to be selected and updated [30].

\section{Input and Output Variables of the Model}

In a certain research, done by Iranian transport research center, the 85th speed and its affecting parameters have been collected for 194 locations [1], so available data lead us to develop a model to determine the previously mentioned parameter using indirect measures. In this research work, new definitions have been introduced to represent the proper variables affecting the 85th speed, which is a part of our novelty in this paper. Input parameters which are three variables are defined corresponding to width, length, and road side condition. Each of them is defined as an integration of its corresponding factors. The 85 th speed has also been categorized into 5 domains based on experimental applications. The suggested process is explained in four stages as follow.

3.1. Defining Integrated Factors and Parameters. As mentioned before, input variables have been categorized into three main integrated factors including width, length, and roadside land use, while each integrated factor consists of a weighted sum of the corresponding parameters as follows:

(i) width parameters:

(a) pavement width,

(b) shoulder width,

(ii) length parameters:

(a) percentage of forbidden overtaking,

(b) number of access points,

(iii) roadside land use parameters:

(a) type of land use (none, residential, industrial, agricultural),

(b) condensation (poor, medium, condensed).

Some of the previous parameters are measurable or quantitative and some are not. For example, the amount of pavement width is measureable and the amount of road side facilities is not. We need to convert all of the input parameters to fuzzy variables. Some of parameters such as pavement width have a proportional effect on 85 th speed that increase maneuverability and speed. On the contrary, other parameters, such as number of access points, have inverse effect on 85th speed which reduce the maneuverability and speed. The previously-mentioned integrated factors, the related parameters, and their effects are shown in Table 1 in which the corresponding symbols and their effects are indicated. 
TABLE 1: The integrated factors and corresponding parameters, types (crisp or fuzzy), symbols, and type of effect on the 85 th speed value.

\begin{tabular}{lcccc}
\hline Integrated factor & Parameter & $\mathrm{C} / \mathrm{F}^{*}$ & Symbol & Effect \\
\hline \multirow{2}{*}{ Width } & Pavement width & $\mathrm{C}$ & $\mathrm{PW}$ & Direct \\
& Shoulder width & $\mathrm{C}$ & $\mathrm{SW}$ & Direct \\
Length & Overtaking & $\mathrm{C}$ & $\mathrm{OV}$ & Indirect \\
& Access point & $\mathrm{C}$ & $\mathrm{AP}$ & Indirect \\
Roadside & Type & $\mathrm{F}$ & $\mathrm{TF}$ & Direct \\
Land use & Condensation & $\mathrm{F}$ & $\mathrm{CF}$ & Direct \\
\hline
\end{tabular}

${ }^{*}$ C: crisp, F: fuzzy.

TABLE 2: Rules for defining the fuzzy variable of roadside facilities.

\begin{tabular}{lcc}
\hline Type & Condensation & LU \\
\hline Without & - & 5 \\
Residential & Poor & 4 \\
Residential & Medium & 3 \\
Residential & Condensed & 2 \\
Industrial & Poor & 4 \\
Industrial & Medium & 3 \\
Industrial & Condensed & 2 \\
Agricultural & Poor & 5 \\
Agricultural & Medium & 4 \\
Agricultural & Condensed & 3 \\
\hline
\end{tabular}

3.2. Defining Fuzzy Variables. For different types and condensations of roadside land use (LU), a number is associated as shown in Table 2. Variable LU represents a combination of both type and condensation of land use in order to be used as input variable. The boundary of each combination has been considered as variable in representation of the fuzzy variable in Table 2, so if a combination of more types of land use or condensation needs to be considered, a simple calculation is proposed to outline the final amount of variable. For example, if roadside land use consists of 20 percent industrial and 80 percent agricultural and condensation is medium, thus variable is calculated by $(0.2 \times 3+0.8 \times 4=3.8)$.

3.3. Converting Parameters to Fuzzy Input Variables. Integrated variables, which represent land use, width, and length including their parameters, are defined according to (6), (7), and (8), respectively. New variables of $V 1, V 2$, and $V 3$ with closed interval of [ $\left[\begin{array}{ll}0 & 1\end{array}\right]$ are considered as inputs to the fuzzy model

$$
\begin{aligned}
& V 1=\frac{W 1 \times \mathrm{LU}}{5} \quad W 1=\left[\begin{array}{ll}
0 & 1
\end{array}\right], \\
& V 2=\frac{W 2 \times P W+W 3 \times S W}{7} \quad W 2, W 3=\left[\begin{array}{ll}
0 & 1
\end{array}\right], \\
& V 3=\frac{(1 /(W 4 \times O V+1))+(1 /(W 5 \times A P+1))}{2} \\
& W 4, W 5=\left[\begin{array}{ll}
0 & 1
\end{array}\right] .
\end{aligned}
$$

$W 1, \ldots, W 5$ weighting factors are considered to be used by the process of particle swarm optimization. New variables
TABLE 3: Normalized variables of factors by fuzzy sets.

\begin{tabular}{lcccc}
\hline State & Bound & $\begin{array}{c}\text { Land use } \\
(V 1)\end{array}$ & $\begin{array}{c}\text { Width } \\
(V 2)\end{array}$ & $\begin{array}{c}\text { Length } \\
(V 3)\end{array}$ \\
\hline 0 & $0-0.25$ & Condensed & Narrow & $\begin{array}{c}\text { Low } \\
\text { maneuverable } \\
\text { Medium }\end{array}$ \\
0.5 & $0.25-0.75$ & Medium & Medium & $\begin{array}{c}\text { Maneuverable } \\
\text { High }\end{array}$ \\
1 & $0.75-1$ & Poor & Vast & $\begin{array}{c}\text { Higurable } \\
\text { maneuverable }\end{array}$ \\
\hline
\end{tabular}

TABle 4: Categories (Domains) of 85th speed.

\begin{tabular}{lccc}
\hline State & Fuzzy definition & $\begin{array}{c}\text { Lower bound } \\
(\mathrm{Km} / \mathrm{h})\end{array}$ & $\begin{array}{c}\text { Upper bound } \\
(\mathrm{Km} / \mathrm{h})\end{array}$ \\
\hline 1 & Very low & 51 & 60 \\
2 & Low & 61 & 75 \\
3 & Medium & 76 & 90 \\
4 & High & 91 & 100 \\
5 & Very high & 101 & 110 \\
\hline
\end{tabular}

$V 1, V 2$, and $V 3$, shown in Table 3, are defined as uniformed fuzzy variables when they have been converted to the fuzzy norms. The formulation of PSO provides an ability to meet the previous constraints, while the feasible solutions are kept while parameters are restricted to closed interval [0 1 1].

3.4. Setting Output Variable. The 85th speed is categorized according to instruction manuals, which are being used for road maintenance and road construction building [34]. In this manual, 85 th speed is defined in six domains started from 0 ended to $110 \mathrm{~km} / \mathrm{h}$; however, in this research work the range of speed was considered from 50 to $110 \mathrm{~km} / \mathrm{h}$, and the scope of 85th speed is limited to five classes as shown in Table 4.

\section{Developing PSO-Fuzzy Model}

4.1. Main Structure. The proposed PSO-fuzzy model includes two main parts that are PSO and fuzzy parts. The fuzzy model estimates 85 th speed based on input variables $V 1, V 2$, and $V 3$ while PSO generates input variables based on weighting factors and compares estimated results with corresponding experimental data. The process is an iteration-based one. It means that parameters are updated in each sequence of running the model by checking stopping criteria. The stopping criterion is a constant number of iterations.

A PSO-fuzzy model is required to go through the following steps:

(1) initialization of weighting parameters $(W 1, \ldots, W 5)$;

(2) converting crisp variables to fuzzy ones using (6), (7), and (8);

(3) running the fuzzy part of the model;

(4) checking stopping criteria; 


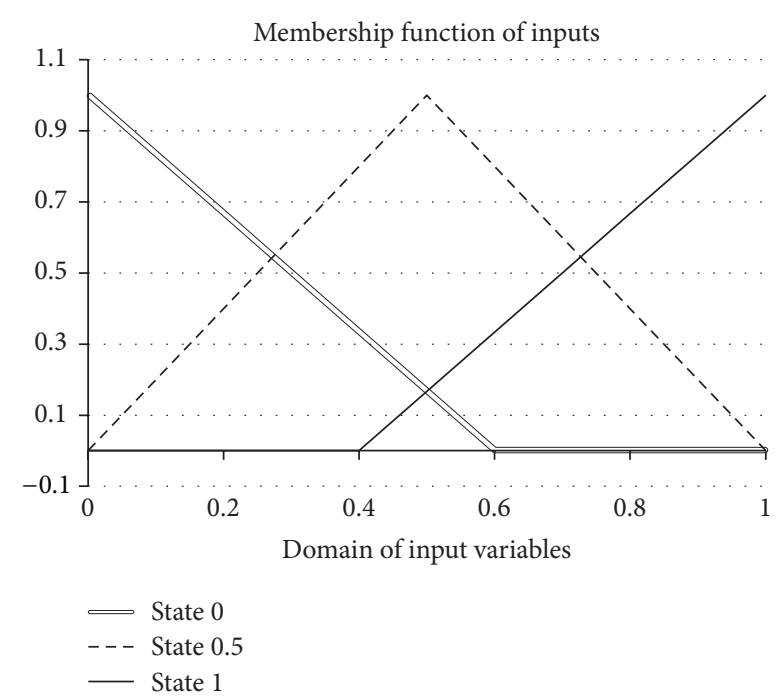

FIgURE 2: Membership functions corresponding to input variables.

(5) if stopping criteria are met, go to step (7) and show results;

(6) update weighting parameters according to PSO velocity and movement, and then go to step (2);

(7) check and compare results with another method.

4.2. Defining Membership Functions and Fuzzy Model. The first step of developing the fuzzy model is to define membership functions corresponding to input and output variables. Input variables are defined by one-interval domain, so a triangular membership function is appropriate to define input variables. Output variable is defined into five categories, so a trapezoidal membership function is used to define output variable. For each of the three input variables, a triangular membership functions is defined [11]. Figure 2 shows a sample of membership function utilized for input variables. A trapezoidal membership function is used for output variable as shown in Figure 3. The overall view of fuzzy model is also shown in Figure 4.

4.3. Rule Definition. For this fuzzy model, the corresponding rules are defined according to the traffic experts' points of view which explain the relationships between input and output variables [11]. Since the road width, road length, and land use have three different states, a maximum number of $3 \times 3 \times 3=27$ rules can be used to define the relationship between input and output variables. The corresponding states of the rules are shown in Table 5.

4.4. Model Presentation. Minimizing the number of incorrect assignment for defined categories of 85 th speed is the main objective function in the proposed PSO-fuzzy model. Assume that $N_{m i}$ is the number of samples assigned to the $i$ th category

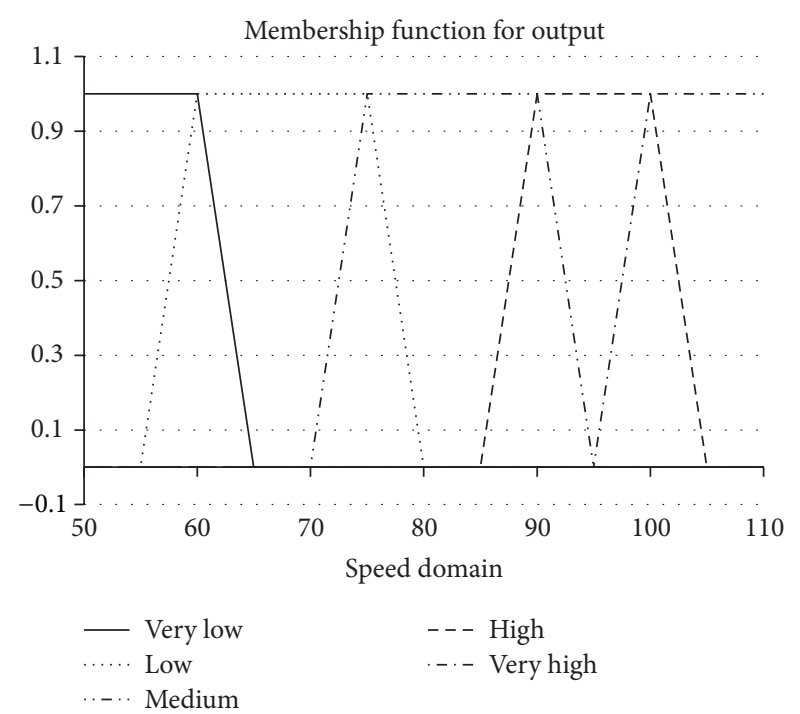

FIGURE 3: Membership function corresponding to 85 th speed.

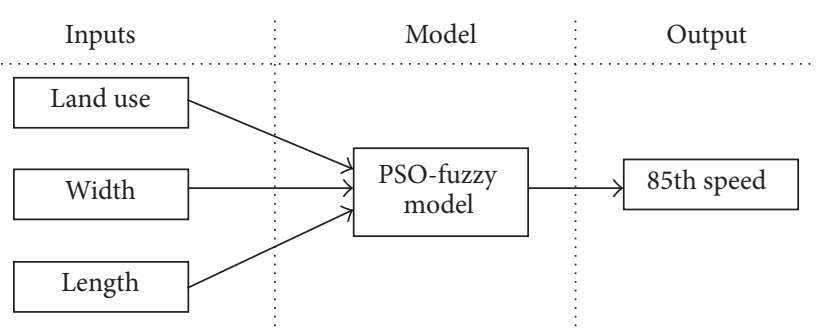

Figure 4: Overall view of fuzzy model.

by model and $N_{o i}$ is the observed one; then objective function would be defined according to the following equation:

$$
\begin{aligned}
\text { Min } Z= & \sum_{i=1}^{5}\left|N_{m i}-N_{o i}\right| \\
& \text { for } i=1, \ldots, 5 \text { (all speed categories) }
\end{aligned}
$$

Subject to: model constraints,

where, new variables defined by (6) to (8) will calculate the normalized variables which are assigned to the 85 th speed categories:

$$
\begin{gathered}
N m i=\operatorname{Sum}(\text { Rule }(V 1, V 2, V 3), i) \text { for } i=1, \ldots, 5, \\
V 1=\frac{W 1 \times \mathrm{LU}}{5}, \\
V 2=\frac{W 2 \times P W+W 3 \times S W}{7}, \\
V 3=\frac{(1 /(W 4 \times O V+1))+(1 /(W 5 \times A P+1))}{2} .
\end{gathered}
$$

And model constraints are as follows:

$$
\begin{gathered}
0 \leq V 1, V 2, V 3 \leq 1, \\
0 \leq W 1, W 2, W 3, W 4, W 5 \leq 1 .
\end{gathered}
$$


TABLE 5: Rule definition for 85th speed fuzzy model.

\begin{tabular}{|c|c|c|c|c|}
\hline Rule & Land use & Width & Length & Speed \\
\hline 1 & 0 & 0 & 0 & 1 \\
\hline 2 & 0 & 0 & 0.5 & 1 \\
\hline 3 & 0 & 0 & 1 & 2 \\
\hline 4 & 0 & 0.5 & 0 & 1 \\
\hline 5 & 0 & 0.5 & 0.5 & 2 \\
\hline 6 & 0 & 0.5 & 1 & 3 \\
\hline 7 & 0 & 1 & 0 & 2 \\
\hline 8 & 0 & 1 & 0.5 & 2 \\
\hline 9 & 0 & 1 & 1 & 3 \\
\hline 10 & 0.5 & 0 & 0 & 2 \\
\hline 11 & 0.5 & 0 & 0.5 & 2 \\
\hline 12 & 0.5 & 0 & 1 & 3 \\
\hline 13 & 0.5 & 0.5 & 0 & 2 \\
\hline 14 & 0.5 & 0.5 & 0.5 & 2 \\
\hline 15 & 0.5 & 0.5 & 1 & 3 \\
\hline 16 & 0.5 & 1 & 0 & 3 \\
\hline 17 & 0.5 & 1 & 0.5 & 4 \\
\hline 18 & 0.5 & 1 & 1 & 4 \\
\hline 19 & 1 & 0 & 0 & 3 \\
\hline 20 & 1 & 0 & 0.5 & 4 \\
\hline 21 & 1 & 0 & 1 & 4 \\
\hline 22 & 1 & 0.5 & 0 & 4 \\
\hline 23 & 1 & 0.5 & 0.5 & 5 \\
\hline 24 & 1 & 0.5 & 1 & 5 \\
\hline 25 & 1 & 1 & 0 & 4 \\
\hline 26 & 1 & 1 & 0.5 & 5 \\
\hline 27 & 1 & 1 & 1 & 5 \\
\hline
\end{tabular}

Rule function in (10) is the result of fuzzy function derived by Table 5 . In the proposed model $W 1, \ldots, W 5$ are PSO parameters which should be estimated and are restricted in closed interval $\left[\begin{array}{ll}0 & 1\end{array}\right]$. Closed interval [ $\left[\begin{array}{ll}0 & 1\end{array}\right]$ should be met in order to keep feasibility solutions. Other parameters are defined in Table 1. Iterative procedure of PSO calculates the amount of each parameter in iterations and converge them to the best solution. A full model of PSO $(\varphi 1, \varphi 2>0)$ has been developed because Gbest (global optimum) and Pbest (population optimum) are required to be involved in velocity calculation.

Because the convergence speed in the particle swarm optimization algorithm with the constriction coefficient (convergence agent) is much quicker than inertia factor [30], constriction coefficient by a different structure from conventional one is used to control velocities via (15) and (16):

$$
\begin{aligned}
V i 5=\operatorname{Max} & (0, V i 5+\varphi 1 \times \operatorname{random} \times(P i 5-W i 5)+\varphi 2 \\
& \times \operatorname{random} \times(P g 5-X i 5)) \quad \text { if step }<0,
\end{aligned}
$$

$$
\begin{aligned}
V i 5=\operatorname{Min} & (1, V i 5+\varphi 1 \times \operatorname{random} \times(P i 5-W i 5)+\varphi 2 \\
& \times \operatorname{random} \times(P g 5-X i 5)) \quad \text { if step }>0,
\end{aligned}
$$

where,

$$
\begin{aligned}
\text { Step }= & (\varphi 1 \times \text { random } \times(P i d-X i d) \\
& +\varphi 2 \times \text { random } \times(P g d-X i d)) .
\end{aligned}
$$

A fully connected neighborhood topology has been used as swarm topology in the model; therefore all particles have been connected to each other. All particles are updated in each iteration using synchronous method of updating.

\section{Running PSO-Fuzzy Model}

5.1. Parameter Calculation and Discussion. A set of 101 experimental data has been used to adjust the PSO-fuzzy model. Data have been practically gathered for speed zone determination in another research work [1], but they are available for checking the proposed algorithm. Although a lot of variables have been gathered in the above research work, the related attributes are used to check the performance of proposed algorithm. The objective function is the accuracy of the model and the percentage of correct assignment of the speed values to the whole observations is defined as accuracy of the model. The error corresponding to the input set of data has been reduced by changing the weighing factors as optimization variables. The number of iterations and population size are two main parameters affecting the improvement of the model. They have significant roles in finding the best values for weighing factors. For each population size and iteration number, the means of the results corresponding to five times of running are shown in Table 6 . This table shows that the population size of 100 and the iteration number of 200 to 500 are sufficient for an accuracy of $96 \%$. No more improvement has been observed when the numbers of iteration and population size are increased. Results also revealed that the population size and iteration number dramatically change the parameters, and accuracy. It means that using PSO may be a good technique for parameter tuning in these kinds of objective functions.

5.2. Comparison with Exponential Model. In order to compare the accuracy of results, a conventional method of exponential regression model for parameter estimation has been used. In this model, if the effects of independent variables on dependent variable are power based, the model is called exponential regression. Parameters are calculated based on the minimum of mean or total square errors between observations and model outputs [35]. In this model, independent variables $X_{1}, X_{2}, \ldots, X_{n}$ and dependent variable $Y$ are estimated by the following equation:

$$
Y=\beta_{0} \times \exp \left(\beta_{1} X_{1}\right) \times \exp \left(\beta_{2} X_{2}\right) \times \cdots \times \exp \left(\beta_{n} X_{n}\right) .
$$

In this equation, coefficients $\beta_{0}, \beta_{1}, \beta_{2}, \beta_{3}, \ldots$, and $\beta_{n}$ are estimated through minimization of mean or total square 
TABLE 6: Results of running PSO-fuzzy model of 85th speed.

\begin{tabular}{|c|c|c|c|c|c|c|c|}
\hline \multirow{2}{*}{ Population size } & \multirow{2}{*}{ Iteration number } & \multicolumn{5}{|c|}{ Parameters } & \multirow{2}{*}{ Accuracy (percent) } \\
\hline & & $W 1$ & $W 2$ & $W 3$ & W4 & $W 5$ & \\
\hline \multirow{3}{*}{20} & 100 & 0.623 & 0.725 & 0.302 & 0.498 & 0.423 & 91 \\
\hline & 200 & 0.615 & 0.749 & 0.294 & 0.514 & 0.427 & 93 \\
\hline & 500 & 0.684 & 0.771 & 0.286 & 0.519 & 0.408 & 94 \\
\hline \multirow{3}{*}{50} & 100 & 0.649 & 0.789 & 0.246 & 0.515 & 0.424 & 93 \\
\hline & 200 & 0.632 & 0.779 & 0.244 & 0.512 & 0.418 & 94 \\
\hline & 500 & 0.682 & 0.808 & 0.254 & 0.567 & 0.434 & 95 \\
\hline \multirow{3}{*}{100} & 100 & 0.598 & 0.805 & 0.213 & 0.532 & 0.402 & 95 \\
\hline & 200 & 0.610 & 0.817 & 0.204 & 0.543 & 0.398 & 96 \\
\hline & 500 & 0.622 & 0.844 & 0.212 & 0.569 & 0.389 & 96 \\
\hline
\end{tabular}

errors between observations and model outputs [36]. Parameters have been estimated for experimental data using solver option in Microsoft Excel and model has been formulated as (19):

$$
\begin{aligned}
\text { Speed }= & 110.028 \times e^{0.0899 P W} \times e^{0.00295 S W} \times e^{-0.0991 O V} \\
& \times e^{-0.0160 A P} \times e^{0.0596 T R} \times e^{-0.0845 C R} .
\end{aligned}
$$

The accuracy of exponential model was $84 \%$, while that of proposed PSO-fuzzy model was $96 \%$ which reveals that PSOfuzzy model is a more accurate model to estimate 85 th speed of vehicles in intercity roads. There are two main reasons to achieve better accuracy. The first is using new variables defined as $V 1, V 2$, and $V 3$ in Section 3.3 and the second is developing a nonlinear structures which are utilized in other techniques such as neural network approved in other studies [37].

\section{Summary and Conclusion}

In this paper, a PSO-fuzzy model has been developed and utilized to determine the category of the 85th speed of vehicles in undivided intercity roads. Roadside facilities (land use), width, and length characteristics of roads are considered as input variables and the category of 85 th speed as output variable to the fuzzy model, respectively. New definitions of input variables for fuzzy model have been proposed to combine crisp and fuzzy variables in the model. Input variables were converted to normalized format using weighting factors, which are estimated by particle swarm optimization technique. Fully neighborhood particles are considered as topology, while constriction coefficient is used for velocity limitation in synchronous method of updating particles.

A set of 101 experimental data has been used for tunning the weighting factors (PSO parameters). Results show that the algorithm of combining PSO and Fuzzy as well as and new definition of input variables integrating with the crisp and fuzzy variables is capable of estimating the category of 85 th speed of the road passing vehicles. The developed model is capable of estimating the 85th speed with a high accuracy of $96 \%$, while conventional method of exponential regression estimates speed categories corresponding to the experimental data with an accuracy of $84 \%$.
Results also reveal that the model is sensitive to population size and iteration number which are basically used by PSO algorithm. As population size and iteration number became larger, better results are achieved. Land use parameter and pavement width represented by weighting factor $W 1$ and $W 2$, respectively, have significant effect on 85 th speed. The percentage of forbidden overtaking represented by $W 4$ has indirect effect on 85th speed. However, its effect is not as large as the effects of the land use and pavement width. Shoulder width and number of access points, represented by $W 3$ and $W 5$, respectively, have less effect on 85 th speed. It also shows that pavement width is more important to drivers' speed than shoulder width as well as the number of access points plays a less significant role on 85th speed.

For future researches, it is recommended to develop a more complicated fuzzy model in order to apply more input variables such as awareness campaigns and enforcement activities. In addition, the developed fuzzy model may be utilized for all types of the roads particularly for divided roads and freeways.

\section{References}

[1] Transportation Research Institute Andishe Rahian Gostaresh, "Calibration of zoning speed limit in rural roads," Report no. 1, 2007.

[2] G. A. Rezai Rad and J. Mohamadi, "Vehicle speed estimation based on the image," in Proceedings of the 4th International Conference: Sciences of Electronic, Technologies of Information and Telecommunications, pp. 1-5, 2007, Hammamet, Tunisia, March 2007.

[3] A. Hussain, K. Shahzad, and C. Tang, "Real time speed estimation of vehicles," World Academy of Science, Engineering and Technology, vol. 61, pp. 726-730, 2012.

[4] L. H. Martin, "Estimating vehicle speed from traffic count and occupancy data," Journal of Data Science, vol. 2, pp. 231-244, 2004.

[5] S. I. Manjari Gupta and A. Bhattacharyya, "A study on lateral speed estimation methods," International Journal of Vehicle Autonomous Systems, vol. 2, no. 1-2, 2004.

[6] D. Bauer, P. Bühler, N. Donath et al., "Embedded vehicle counting system with "silicon retina" optical sensor," in Workshop on Information Optics, Toledo, Spain, 2006. 
[7] N. Kasem, A. Kosba, and M. Youssef, "An RF-based vehicle detection and speed estimation system," in Proceedings of the 75th IEEE Vehicular Technology Conference (IEEE VTC '12), Yokohama, Japan, 2012.

[8] Y. U. Ali, P. Huei, and H. E. Tseng, "A study on lateral speed estimation methods," International Journal of Vehicle Autonomous Systems, vol. 2, no. 1-2, pp. 126-144, 2004.

[9] P. Augural, Fuzzy Model for Estimating of Passenger Car Unit, National Institute of Technology, Information Science and Application, Kurukshetra, India, 2008.

[10] P. Panetta, J. Poj, and N. Nanokchai, Evaluation of Road Traffic Congestion Using Fuzzy Techniques, King Mongkut South University of Technology, Thonburi, Thailand, 2008.

[11] P. Pongpaibool, P. Jangmchil, and N. Noodwong, Evaluation of Road Traffic Congestion Using Fuzzy Techniques, King Mongkut South University of Technology, Thonburi, Thiland, 2008.

[12] L. Ruey-Hsun, T. Sheng-Ren, C. Yie-Tone, and T. Wan-Tsun, "Optimal power flow by a fuzzy based hybrid particle swarm optimization approach," Electric Power Systems Research, vol. 81, no. 7, pp. 1466-1474, 2011.

[13] L. Wang and C. Singh, "Balancing risk and cost in fuzzy economic dispatch including wind power penetration based on particle swarm optimization," Electric Power Systems Research, vol. 78, no. 8, pp. 1361-1368, 2008.

[14] S. Manna, R. H. Kenneth, M. El-Halwagi, R. Longari, and M. Gentile, Development of Hierarchical Fuzzy Model for the Evolution of Inherent Safety, Universidad de las Americas, Pueblea, Mexico, 2004.

[15] C. Yang, S. Fan, Z. Wang, and W. Li, "Application of fuzzy control method in a tunnel lighting system," Mathematical and Computer Modelling, vol. 54, no. 3-4, pp. 931-937, 2011.

[16] S. Hoogendoorn, S. L. Hoogendoorn, and H. Schuurman, Fuzzy Perspective in Traffic Engineering, Delft university of technology and Ministry of Transport, Delft, The Netherlands, 2000.

[17] Y. Lu, J. Hinze, and Q. Li, "Developing fuzzy signal detection theory for workers' hazard perception measures on subway operations," Safety Science, vol. 49, no. 3, pp. 491-497, 2011.

[18] E. Bagheri, M. Feizi, F. Enson, and F. Behnia, Novel Fuzzy Control Model of Traffic Light Timing at an Urban Intersection, Department of Computer Science, University of New Branswick, Prediction, Canada, 2004.

[19] J. L. Castro, M. Delgado, J. Medina, and M. D. Ruiz-Lozano, "An expert fuzzy system for predicting object collisions. Its application for avoiding pedestrian accidents," Expert Systems with Applications, vol. 38, no. 1, pp. 486-494, 2011.

[20] G. Ficili and D. Panno, "A fuzzy algorithm for combined control of traffic parameters: assessment and key issues," Computer Communications, vol. 22, no. 3, pp. 199-210, 1999.

[21] C. F. Juang and C. Y. Wang, "A self-generating fuzzy system with ant and particle swarm cooperative optimization," Expert Systems with Applications, vol. 36, no. 3, pp. 5362-5370, 2009.

[22] I. H. Kuo, S. J. Horng, T. W. Kao, T. L. Lin, C. L. Lee, and Y. Pan, "An improved method for forecasting enrollments based on fuzzy time series and particle swarm optimization," Expert Systems with Applications, vol. 36, no. 3, pp. 6108-6117, 2009.

[23] H. Liu, A. Abraham, and A. E. Hassanien, "Scheduling jobs on computational grids using a fuzzy particle swarm optimization algorithm," Future Generation Computer Systems, vol. 26, no. 8, pp. 1336-1343, 2010.

[24] Y. L. Huang, S. J. Horng, M. He et al., "A hybrid forecasting model for enrollments based on aggregated fuzzy time series and particle swarm optimization," Expert Systems with Applications, vol. 38, no. 7, pp. 8014-8023, 2011.

[25] F. Valdez, P. Melin, O. Castillo, and O. Montiel, "A new evolutionary method with a hybrid approach combining particle swarm optimization and genetic algorithms using fuzzy logic for decision making," in Proceedings of the IEEE Congress on Evolutionary Computation (CEC '08), pp. 1333-1339, June 2008.

[26] A. Robati, G. A. Barani, H. Nezam Abadi Pour, M. J. Fadaee, and J. Rahimi Pour Anaraki, "Balanced fuzzy particle swarm optimization," Applied Mathematical Modelling, vol. 36, no. 5, pp. 2169-2177, 2012.

[27] L. Zhao, F. Qian, Y. Yang, Y. Zeng, and H. Su, "Automatically extracting T-S fuzzy models using cooperative random learning particle swarm optimization," Applied Soft Computing Journal, vol. 10, no. 3, pp. 938-944, 2010.

[28] F. Valdez, P. Melin, and O. Castillo, "An improved evolutionary method with fuzzy logic for combining Particle Swarm Optimization and Genetic Algorithms," Applied Soft Computing Journal, vol. 11, no. 2, pp. 2625-2632, 2011.

[29] J. Kennedy and R. Eberhart, "Particle swarm optimization," in Proceedings of the IEEE International Conference on Neural Networks, vol. 4, pp. 1942-1948, December 1995.

[30] Q. Bai, "Analysis of particle swarm optimization algorithm," Computers and Information Science, vol. 3, no. 1, pp. 180-184, 2010.

[31] J. Kennedy, "Particle swarm: social adaptation of knowledge," in Proceedings of the IEEE International Conference on Evolutionary Computation (ICEC '97), pp. 303-308, IEEE Press, April 1997.

[32] M. Clerc and J. Kennedy, "The particle swarm-explosion, stability, and convergence in a multidimensional complex space," IEEE Transactions on Evolutionary Computation, vol. 6, no. 1, pp. 58-73, 2002.

[33] S. Ghosh, D. Kundu, K. Suresh et al., "On some properties of the lbest topology in particle swarm optimization," in Proceedings of the 9th International Conference on Hybrid Intelligent Systems (HIS '09), pp. 370-375, August 2009.

[34] Iranian Management and Planning Organization, Road Safety Instruction Manual, vol. 3-7, no. 267, 1st edition, 2005.

[35] P. G. Hoel, Elementary Statistics, John Wiley and Sons, New York, NY, USA, 4th edition, 1976.

[36] G. A. F. Seber and C. J. Wild, Nonlinear Regression, John Wiley \& Sons, New York, NY, USA, 1989.

[37] A. Mahmoudabadi, "Comparison of weighted and simple linear regression and artificial neural network models in freeway accidents prediction," in Proceeding of the 2nd International Conference on Computer and Network Technology, pp. 392-396, Bangkok, Thailand, April 2010. 


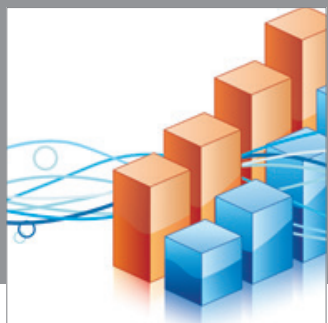

Advances in

Operations Research

mansans

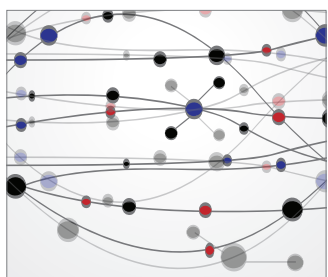

The Scientific World Journal
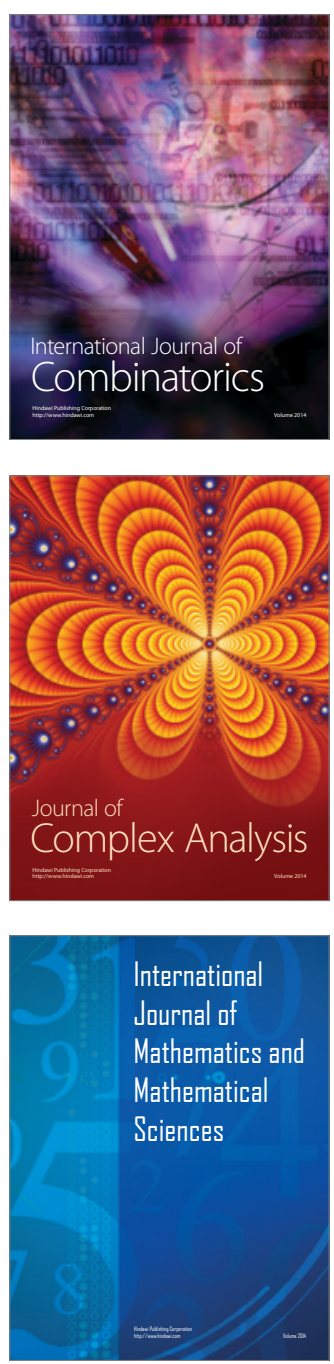
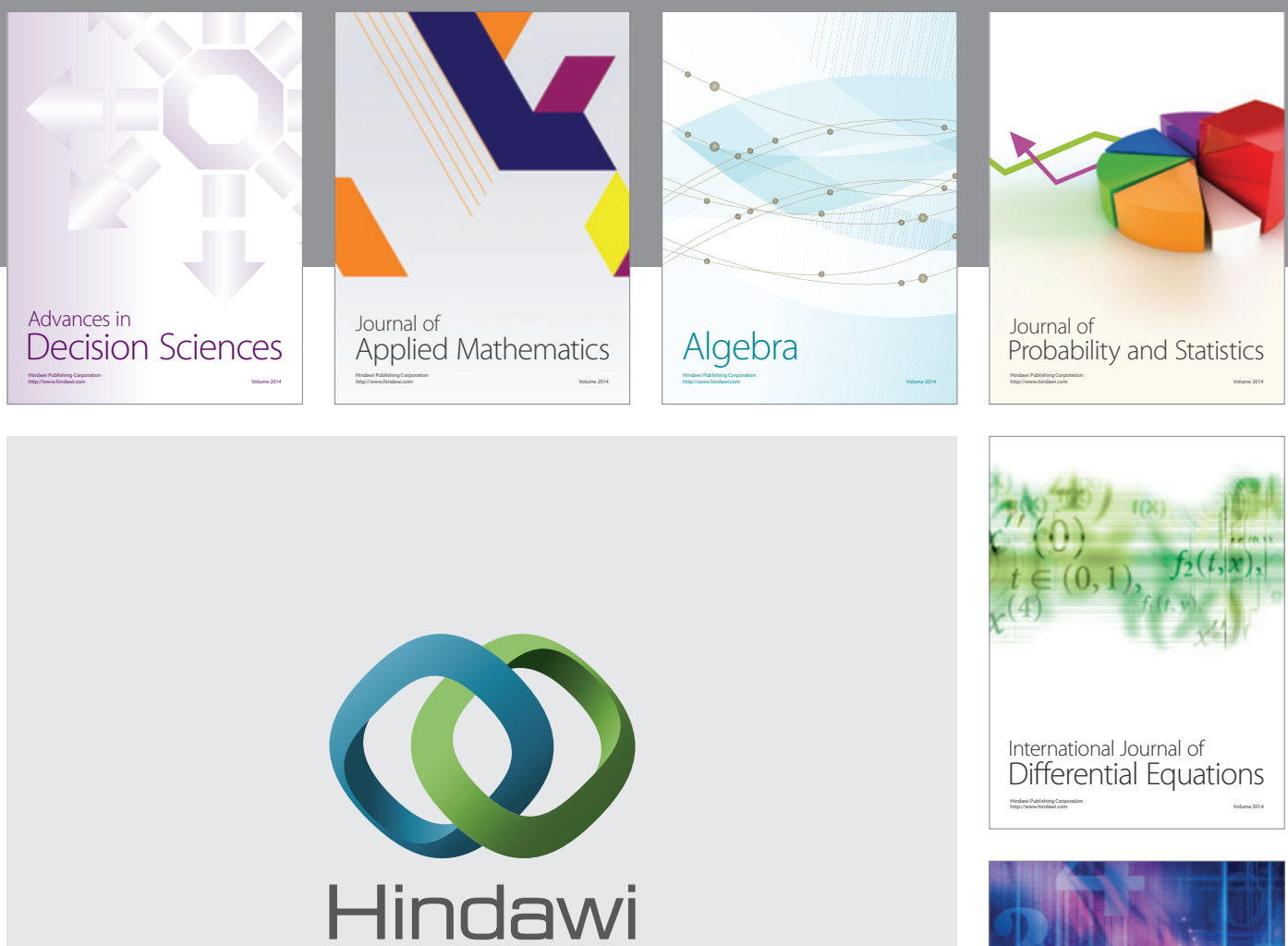

Submit your manuscripts at http://www.hindawi.com
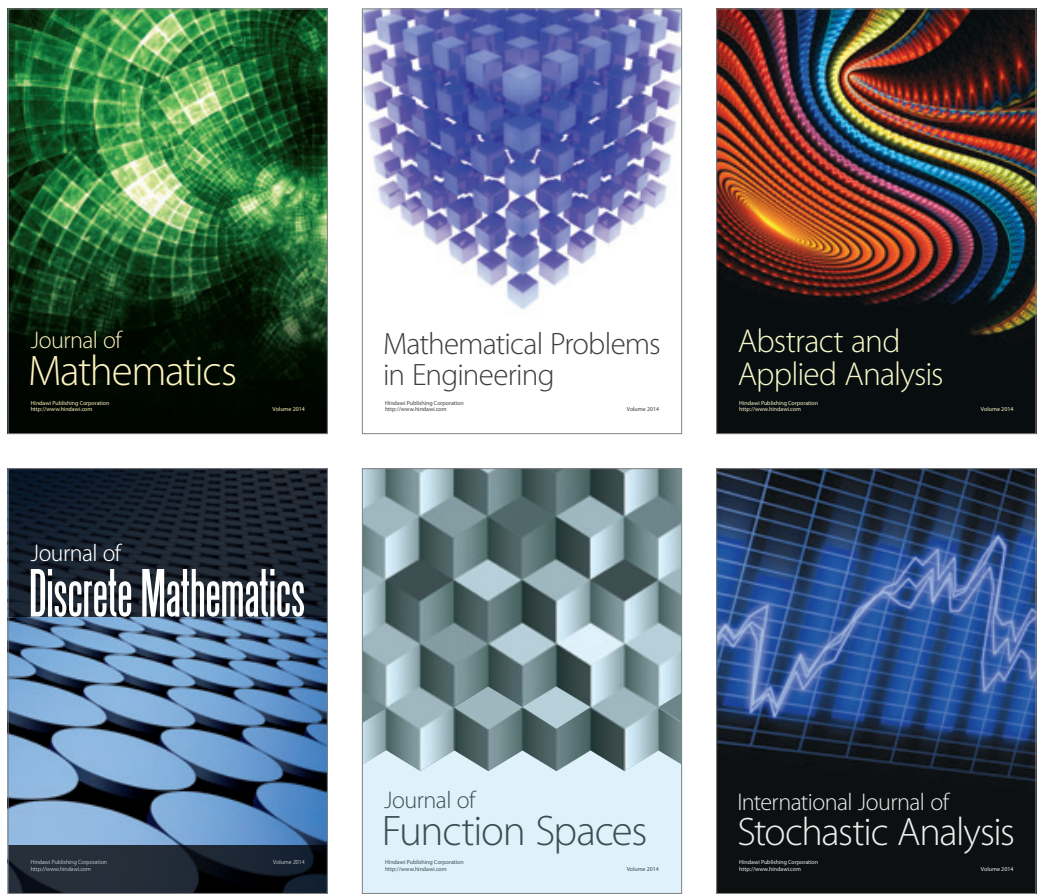

Journal of

Function Spaces

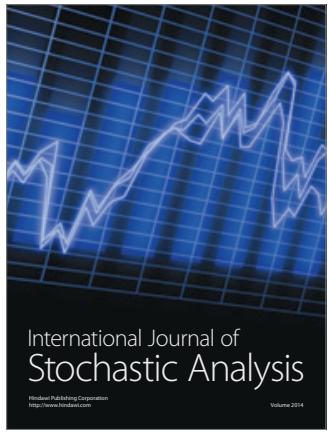

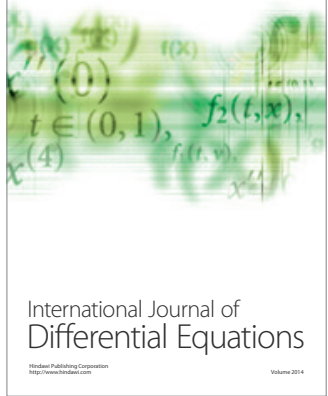
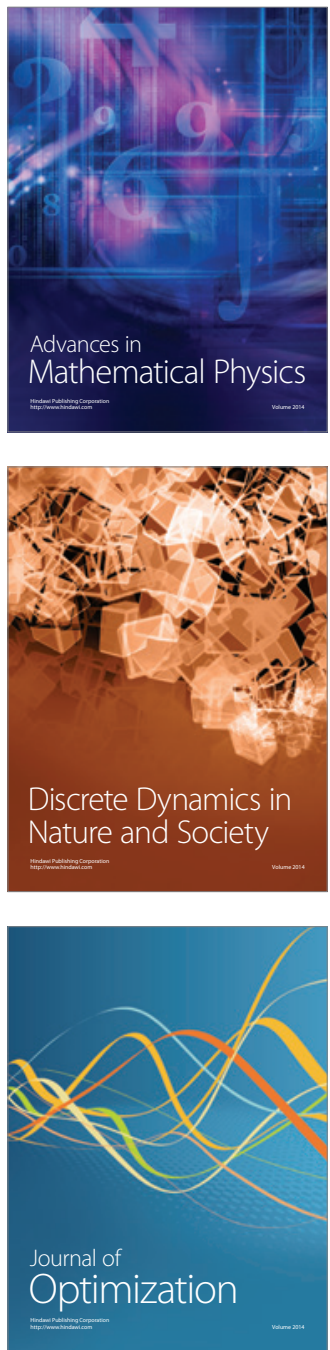